JAMA Facial Plastic Surgery I Original Investigation

\title{
Evaluation of a Vertical Box Plating Technique for Mandibular Body Fractures and Retrospective Analysis of Patient Outcomes
}

Daniel Demesh, MD; James A. Leonard, BS; Clyde B. Schechter, MD; Prince Dhillon, DMD, MD; Wayne Hsueh, MD; Howard Stupak, MD

IMPORTANCE Despite advancements, treatment of mandibular body fractures is plagued by complications. Evaluation of a new plating system is needed with the goal of reducing complication rates.

OBJECTIVES To evaluate the biomechanical behavior of a vertically oriented box plate vs traditional rigid internal fixation plating techniques for mandibular body fractures and to test if placement of the 3-dimensional plate oriented parallel to the fracture line provides improved rigidity and greater resistance to torsion, resulting in improved outcomes.

DESIGN, SETTING, AND PARTICIPANTS A mandible fracture model with synthetic replicas was used to compare resistance to torsional forces of different plating configurations. Additionally, a retrospective comparative review of the medical records of 84 patients with mandibular body fractures treated from 2005 to 2018 at Jacobi Medical Center, a level-1 trauma hospital in Bronx, New York, was completed.

EXPOSURES Patients sustained a mandibular body fracture and were treated with open reduction and internal fixation using metal plating.

MAIN OUTCOMES AND MEASURES In the comparative study of biomechanical behavior of various plating configurations, maximum torque sustained prior to deformation and loss of alignment was measured. Medical records were reviewed for surgical approach, plating techniques, operative time, length of admission, and rate of complications, including malocclusion, nonunion, infection, neurosensory disturbance, and wound dehiscence.

RESULTS Of the 84 patients included in the retrospective review, 76 (91\%) were men, and the mean (SD) age was 29.7 (12.0) years. During biomechanical analysis, the vertical box plate provided greater stability and $150 \%$ of the resistance against torsional forces when compared with traditional linear plating. In the retrospective review, analysis showed vertical plating was associated with a lower incidence of postoperative neurosensory disturbance (25 [38\%] patients treated with vertical plating vs 0 patients treated with box plating; $P=.002$ ) and a lower risk of any complication (41 [62\%] vs 6 [33\%], respectively; relative risk, $0.54 ; 95 \% \mathrm{Cl}$, $0.27-1.06 ; P=.03$ ). Vertical plating was associated with reduced operative time (134 minutes vs 70 minutes, respectively; $P<.001$ ).

CONCLUSIONS AND RELEVANCE This investigation suggests that vertical box plating is associated with a lower incidence of postoperative complications and reduced operative time compared with traditional plating techniques. The comparative biomechanical component demonstrated that the vertical box plate offered equal or greater resistance to torsional forces. Further studies of greater power and level of evidence are needed to more robustly demonstrate these benefits.

LEVEL OF EVIDENCE 3. 
$\mathrm{T}$ he primary goal of mandible fracture repair remains to establish preinjury occlusion, often with temporary maxillomandibular fixation using arch bars, bone screws, or skeletal wire. ${ }^{1}$ Anatomical reduction of mandibular fragments is also a principal goal achieved using bone clamps, wires, and/or temporary internal fixation devices. The addition of implantable plates can reduce the necessity or time requirement of maxillomandibular fixation. Adequate fixation is of great importance in successful treatment and often includes the use of reconstructive plates.

The mainstay of current use of internal fixation plates involves the placement of these devices at the inferior or superior border perpendicular to the fracture. These plates were designed to prevent segmental distraction forces from causing failure in a 2 -dimensional plane. However, some studies suggest that these plates may provide poor resistance to torsional or twisting forces, especially when there are multiple fracture sites. ${ }^{2}$ In contrast, 3-dimensional, or box, plates use quadrangle geometry to provide additional stability in all dimensions, which offers better resistance against torque forces. ${ }^{3}$

Ellis, ${ }^{2}$ when questioning how much strength is required in a plate to adequately fixate a mandible fracture, concluded that it was these torsional rotational forces that when not stabilized caused failure of fracture healing. An additional advantage of vertically oriented box plates is a smaller dissection field (Figure 1). In order to place inferior border horizontal plates, a wide field of dissection and exploration is required. To place vertically oriented box plates, a narrower field of dissection is required, potentially requiring a shorter operative time and potentially providing better periosteal support to the healing bone. In this study, vertically oriented box plates were biomechanically tested to measure degree of torsion resisted when compared with standard plates. Additionally, clinical data were reviewed to determine if vertically oriented box plates, which have multiple points of resistance to rotational torque forces, resulted in improved operating parameters, including shorter operative time and reduced failure rates.

\section{Methods}

Evaluation of the vertical box plate began with an analysis using biomechanical models. Synthetic mandible replicas (Stryker) were used to evaluate 6 monocortical mandibular body fracture configurations. A handsaw was used to simulate a body fracture, then different plating sets were applied to simulate the repair. Plating techniques of open reduction and internal fixation were performed as described by the Association for the Study of Internal Fixation. ${ }^{4}$ After reconstructive plates were applied, each mandible was subjected to torsional loading applied by a deflection beam torque wrench (Performance Tools) until the construct failed and caused malunion (Supplement). An external hex-headed bolt was also applied to the parasymphyseal region of the mandible for attachment to the torque wrench during force loading (Figure 1). Measurements were recorded for maximum torque sustained prior to deformation and loss of mandible alignment along fracture lines. Examiners were

\section{Key Points}

Question Does a vertically oriented box plate increase resistance to torsional forces and reduce complications in the treatment of mandibular body fractures?

Findings In this comparative study and retrospective medical record review of 84 patients, it was demonstrated that box plating for mandibular body fractures was associated with reduced neurosensory complications and operative time compared with traditional rigid internal fixation plating techniques.

Meaning Vertically oriented box plates may be a superior treatment option for the open reduction and internal fixation of mandibular body fractures.

blinded to the plating configuration while taking measurements, and average torque values were recorded after 5 trials for each configuration.

A complementary examination of the vertically oriented box plate included a retrospective review that examined patient medical records at Jacobi Medical Center, a level 1 trauma center in Bronx, New York, from 2005 to 2018. The study was approved by the Montefiore Medical Center Institutional Review Board, which included a waiver for patient informed consent because the retrospective data were deidentified. Patients were selected if they had a coding in their electronic medical record for repair with open reduction and internal fixation of a mandibular body or parasymphyseal fracture. Patients who were operated on by either the plastic surgery, otorhinolaryngology, or oral and maxillofacial surgery teams were included. All of the vertical box plating applications were performed via an intraoral approach by the otorhinolaryngology team, while the traditional plating techniques were performed by all 3 surgical services. Patients were excluded if they were treated with a closed technique, had prior surgical treatment, or if a different segment of the mandible was fractured. Patients with multiple fractured segments were included. Medical records were reviewed for surgical approach, rate of complications, plating techniques, operative time, length of maxillomandibular fixation, and length of admission. The complications studied included malocclusion, nonunion, infection, neurosensory disturbance, and wound dehiscence. Operative time was defined as the time from when the patient entered the operating room to when they left. Length of admission was defined as number of postoperative days in the hospital.

For the dichotomous outcomes, Pearson $\chi^{2}$ tests were used to test the null hypothesis of equal occurrence probabilities in the 2 groups. For the count of complications, the MannWhitney $U$ test was used. Time in the operating room was also considered an outcome because it affects the cost of providing treatment. The distributions of operative time were displayed as graphs of Kaplan-Meier time-to-event estimates, and the hypothesis of equal operative time distributions was tested with the log-rank test. A multivariate analysis of operative time was carried out using a Cox proportional hazards model, verifying the proportional hazards assumption with Cox-Snell residuals and graphical inspection. 
Figure 1. Repaired Synthetic Mandible Replica and Deflection Beam Torque Wrench

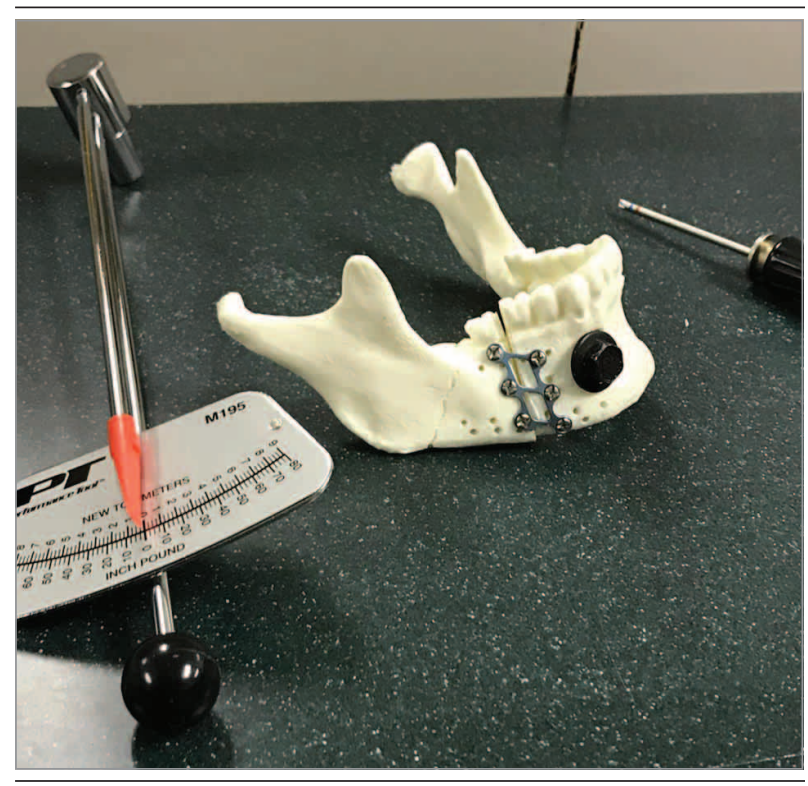

The body fracture in this synthetic mandible replica was repaired using a vertical box plate orientated obliquely along the fracture line. The deflection beam torque wrench used for torque resistance measurement is seen to the left.

\section{Results}

There were 84 distinct patients who accounted for 91 fractures that met eligibility criteria for this study. For patients with multiple fractures, 1 procedure per patient was selected at random for analysis. Of the total patients, 76 (91\%) were men (Table 1). The mean (SD) age was 29.7 (12.0) years. All 8 female patients received the traditional plating technique. Of the 76 men, 18 received the vertical box plating technique. Overall 65 (77\%) patients had multiple mandible fractures, but these additional fractures did not meet study eligibility criteria. The prevalence of such additional fractures was roughly similar in both treatment groups: $83 \%$ ( $n=15$ of 18 ) among those receiving vertical box plating and $76 \%(n=50$ of 66$)$ among those receiving traditional plating.

The otolaryngology team treated 23 (27\%) fractures, 5 with traditional plating and 18 with vertical box plating; the plastic surgery team treated 16 (19\%) fractures, all with traditional plating; and the oral and maxillofacial surgery team treated 45 (54\%) fractures, all with traditional plating. Patients were assigned randomly to surgical services for treatment based on a rotating sequential trauma call pool at the hospital. All 18 patients who received box plating and 27 of the 66 patients who received traditional plating were plated with 1 plate. The remaining 39 patients who received traditional plating required 2 plates. All patients received antibiotics except 2 (11\%) patients who had box plated fractures and $3(5 \%)$ patients who had traditional plated fractures. All 18 patients treated with vertical box plating required an intraoral approach. Of the patients with traditionally plated fractures, 54 (82\%) were treated

\begin{tabular}{ll}
\hline Table 1. Demographics of Study Population & \\
\hline Characteristic & No. (\%) \\
\hline Patient total & 84 \\
\hline Age, mean (SD), y & $29.7(12.0)$ \\
\hline Male & $76(91)$ \\
\hline Patients who received traditional plating & $66(79)$ \\
\hline Prevalence of additional fractures in traditional plating & $50(76)$ \\
\hline Patients who received box plating & $18(21)$ \\
\hline Prevalence of additional fractures in box plating & $15(83)$ \\
\hline
\end{tabular}

intraorally, 5 (8\%) extraorally, and 7 (11\%) with a combined approach.

A disparity was observed in postoperative neurosensory disturbance and occurred in 25 (38\%) patients treated with traditional plating and 0 patients treated with box plating $(P=.002)$ (Table 2). Similarly, measurements of any complication occurred in $41(62 \%)$ patients treated with traditional plating and 6 (33\%) patients treated with box plating (relative risk, $0.54 ; 95 \% \mathrm{CI}, 0.27-1.06 ; P=.03)$. The median number of complications in the traditionally plated group was 1.0 (interquartile range, 2.0) and 0.0 (interquartile range, 1.0) in the vertical box group $(P=.02)$. The rates of postoperative malocclusion, nonunion, reoperation, postoperative infection, facial nerve injury, unfavorable scarring, and wound dehiscence were not individually shown to be lower with any significance based on plating technique.

Differences in operative time were also observed with a median operative time in the traditionally plated group of $134 \mathrm{~min}-$ utes, while the box plated group was 70 minutes $(P<.001)$ (Figure 2). Operative time was further evaluated with a multivariate analysis using a Cox proportional hazards model, verifying the proportional hazards assumption with Cox-Snell residuals and graphical inspection.

In the biomechanical comparative analysis, plating using only a single miniplate resulted in deformation at the fracture line with less than 2.5 inch-pounds ( $<0.28$ newton meters $[\mathrm{N} \cdot \mathrm{m}]$ ) of torque resistance (Table 3 ). Greater resistance was seen using a 6-hole miniplate and a tension band, sustaining forces of $3.75(0.42 \mathrm{~N} \cdot \mathrm{m})$ and 5 inch-pounds $(0.56$ $\mathrm{N} \cdot \mathrm{m}$ ) prior to deformation using a $1-\mathrm{mm}$ and $1.5-\mathrm{mm}$ miniplate, respectively. Up to $150 \%$ greater resistance than single plating was seen using a vertical box plate measuring 7.5 inchpounds $(0.85 \mathrm{~N} \cdot \mathrm{m})$ prior to deformation and a box plate measuring 6.25 inch-pounds $(0.71 \mathrm{~N} \cdot \mathrm{m})$.

\section{Discussion}

Surgical management of mandibular body fractures is still fraught by complications such as malunion, infection, and nerve injury. ${ }^{5}$ A recent article by Ellis ${ }^{2}$ concluded that the most important force causing mandibular fracture complications was the unchecked torsional force that standard horizontal plating only partially addresses. Although that article suggested more rigid plating as a potential solution, further analysis has suggested that multiple vertical points of fixation along a fracture line would more efficiently address the problem. 


\begin{tabular}{lllll}
\hline Table 2. Complications Following Traditional and Vertical Box Plating & & \\
\hline Complication & $\begin{array}{l}\text { Traditional Plate } \\
(\mathrm{n}=66) \text {, No (\%) }\end{array}$ & $\begin{array}{l}\text { Vertical Box Plate } \\
(\mathrm{n}=18), \text { No. (\%) }\end{array}$ & Relative Risk (95\% Cl) & P Value \\
\hline Postoperative malocclusion & $12(18)$ & $2(11)$ & $0.61(0.15-2.49)$ & .48 \\
\hline Nonunion & $4(6)$ & 0 & 0 & .29 \\
\hline Reoperation & $14(21)$ & $5(28)$ & $1.31(0.54-3.15)$ & .56 \\
\hline Postoperative infection & $7(11)$ & 0 & 0 & .15 \\
\hline Neurosensory disturbance & $25(38)$ & 0 & 0 & .002 \\
\hline Facial nerve injury & $2(3)$ & 0 & 0 & .46 \\
\hline Unfavorable scarring & $1(2)$ & 0 & 0 & .60 \\
\hline Wound dehiscence & $4(6)$ & 0 & 0 & .29 \\
\hline Any complication & $41(62)$ & $6(33)$ & $0.54(0.27-1.06)$ & .03 \\
\hline
\end{tabular}

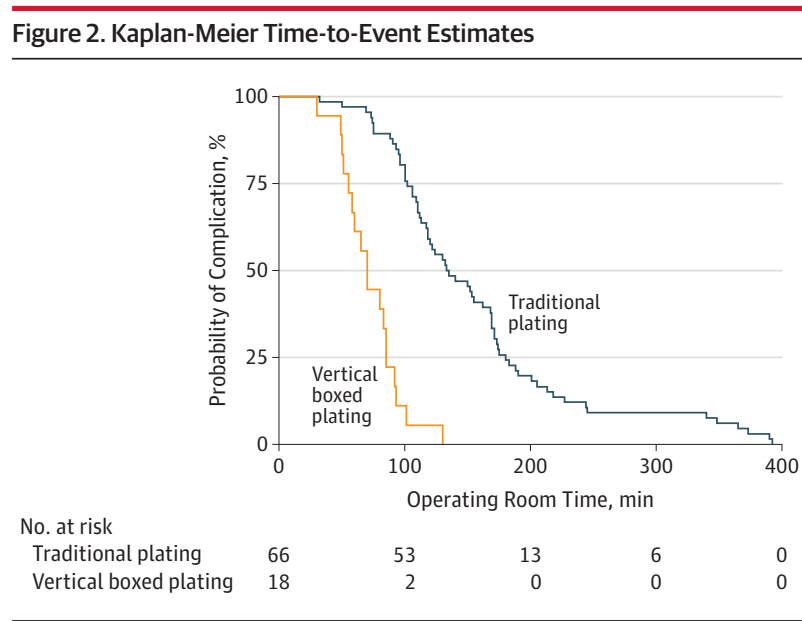

Distributions of operative time are displayed against probability of complications.

Farmand ${ }^{6,7}$ published on his experiences using box plates, which he called 3-D plates, and noted strong resistance to torsional forces, compact form, and lack of major complications. The strength of his plate was based on a geometrically closed quadrangular shape providing a stable configuration in 3 dimensions, not by its thickness or length. Accordingly, we found in a biomechanical fracture model that a plate oriented to provide multiple points of fixation perpendicular to a mandibular body fracture line would better resist torsional forces applied to the fracture. Furthermore, the retrospective clinical study demonstrated lower complication rates and shorter operative time when comparing vertical fixation with conventional techniques.

The biomechanical experiment in the present study demonstrated that the vertical plate was able to provide torque resistance at a level equal to or greater than that of the traditional 2 horizontal plates. Although the traditional plates provided resistance to horizontal distraction, the vertical plate had better torque resistance. The subsequent retrospective review complemented the results from the biomechanical model by demonstrating that the vertically oriented box plate was associated with a lower risk of any complication in the outcomes analysis (62\% vs 33.3\%; $95 \%$ CI, 0.27-1.06; $P=.03$ ). The overall rate of complications in this study was in line with rates that have previously been
Table 3. Torque Sustained Prior to Deformation and Loss of Mandible Alignment Along Fracture Line With Varied Plating Systems

\begin{tabular}{ll}
\hline Plate Configuration Type & $\begin{array}{l}\text { Average Torque } \\
\text { Resistance in } \\
\text { Inch-Pounds }(\mathrm{N} \cdot \mathrm{m})\end{array}$ \\
\hline 1-mm, 4-hole miniplate at superior border & $<2.5(<0.28)$ \\
\hline 1-mm, 6-hole miniplate at inferior border & $<2.5(<0.28)$ \\
\hline $\begin{array}{l}\text { 1.5-mm, 6-hole compression plate (gold) at } \\
\text { inferior border }\end{array}$ & $<2.5(<0.28)$ \\
\hline $\begin{array}{l}\text { 1-mm, 6-hole miniplate at inferior border with } \\
\text { tension band }\end{array}$ & $3.75(0.42)$ \\
\hline $\begin{array}{l}\text { 1.5-mm, 6-hole compression plate (gold) at } \\
\text { inferior border with tension band }\end{array}$ & $5.00(0.56)$ \\
\hline $\begin{array}{l}\text { 1-mm, 3-dimensional box plate } \\
\text { 1-mm, 3-dimensional vertical box plate }\end{array}$ & $6.25(0.71)$ \\
\hline
\end{tabular}

Abbreviation: $\mathrm{N} \cdot \mathrm{m}$, newton meters.

reported in the literature. ${ }^{8}$ Additionally in the analyses, the vertical box plating technique was also associated with a markedly lower incidence of postoperative neurosensory disturbance. We believe that this may be because of the vertical nature of the plate, which decreases the lateral extent of subperiosteal dissection needed to apply the plate as compared with the larger horizontal plates. As such, less nerve "skeletonization" is required, thereby decreasing the risk to the mental nerve. All of the other rates of individual complication outcomes were lower in the vertical box plate group except for the rate of reoperation, but none met statistical significance. The relative increased rate of reoperation in the vertical box group included a case where a patient requested to have the plates removed as a requirement to serve in the military and a second patient who requested electively to have the plate removed. The study was not sufficiently powered to show a decrease in complications such as malunion and malocclusion independently, though overall the lower rate of any complication in the vertical box group was significant.

The study also demonstrated that placement of the vertical box plate was associated with an decrease in operative time (median time, 133 minutes for traditional plating vs 70 minutes for vertical box plating; $P<.001$ ). This reduction is beneficial not only for the efficiency of the surgeon, but also, importantly, for patient safety and to reduce hospital costs. Although a full cost comparison is outside the scope of this 
study, it must be noted that traditional plating techniques often call for multiple plating configurations while the vertical box is applied as a single plate, which further leads to lower costs.

A review of the literature reveals previous studies with similar conclusions, including a prospective study of 28 patients by Sadhwani and colleagues, ${ }^{9}$ which compared outcomes between 2-mm miniplates and box plates for parasymphyseal fractures. The group with the horizontal miniplates had increased complications, including postoperative mobility, malunion, and infection as compared with the group treated with box plates. Another prospective study by Barde and colleagues ${ }^{3}$ also showed that operative time was significantly reduced with box plating as compared with traditional horizontal plating, but they were unable to show any significant differences in outcomes. In a recent metaanalysis by Wusiman and colleagues ${ }^{10}$ comparing box vs standard miniplates for mandible fractures, the authors were able to show a significant decrease in complication rates with the use of box plates. They included 17 studies, including 9 randomized controlled trials, and demonstrated that the use of box miniplates in the fixation of mandibular fractures decreases the risk of postoperative complications by $52 \%$.

\section{Limitations}

Because this study had both a laboratory and a retrospective medical record review arm, we attempted to minimize the limitations that either study type inherently maintains. However, this combined approach to evaluating a technique still has limitations both clinically and biomechanically. In the laboratory testing, there is limited extrapolation to clinical scenarios owing to the isolated and controlled laboratory setting in which the plating was performed. The experiment tested application of torsion in a single axis (rotating the segments along the axis of the mandible body) while in practice there are certainly multiple angles of force applied at the fracture line. We attempted to limit bias by running each plate configuration 5 times and recording the torque measurements on video. We also blinded the investigators to the plating configuration being tested during calculation of the maximum torque values. The point of maximal torsion was measured just before misalignment at the fracture line based on visual cues, which were subjective.

An intrinsic limitation of this study is that it is a retrospective review and not a randomized clinical trial. A direct comparison of vertical box plating vs traditional plating would require randomization. This study also included a limited sample size. Owing to the fact that the novel plating technique was only used by a single department, there is a possibility of additional confounding variables beyond plate shape. Multiple comparisons were carried out, so there is appreciable risk that these findings constitute type 1 errors. More importantly, the selection of plating technique was strongly associated with patient attributes, surgical team, and other aspects of treatment in this nonrandomized study. The ability to separate the influence of the plating technique from these other influences on outcomes was limited. Our best effort in this regard led to the conclusion that the separate influence of the plating technique on complication rates itself is probably close to zero and could not be estimated precisely enough to establish its direction. In our attempt to limit surgical team bias, both otolaryngology and oral and maxillofacial surgery team members were involved in data collection and analysis. One finding that seems robust is that the vertical plating technique is strongly associated with reduced operative time. This finding remains quite strong even in the multivariate Cox analysis, which also permitted adjustment for surgical team.

\section{Conclusions}

Vertical box plating for mandibular body fractures provided increased torque resistance at the fracture line in biomechanical testing and decreased complications and cost in a retrospective medical chart review compared with traditional plating techniques. Further randomized prospective studies are needed to demonstrate if improved resistance to rotational torsion translates to improvement in clinical outcomes.

\section{ARTICLE INFORMATION}

Accepted for Publication: February 4, 2019.

Published Online: May 23, 2019.

doi:10.1001/jamafacial.2019.0057

Author Affiliations: Department of Otorhinolaryngology-Head and Neck Surgery, Montefiore Medical Center, Albert Einstein College of Medicine, Bronx, New York (Demesh); Albert Einstein College of Medicine, Bronx, New York (Leonard); Department of Epidemiology \& Population Health, Montefiore Medical Center, Albert Einstein College of Medicine, Bronx, New York (Schechter); Department of Oral and Maxillofacial Surgery, Jacobi Medical Center, Albert Einstein College of Medicine, Bronx, New York (Dhillon); Department of Otolaryngology-Head and Neck Surgery, University Hospital, Rutgers New Jersey Medical School, Newark (Hsueh); Department of Otorhinolaryngology-Head and
Neck Surgery, Jacobi Medical Center, Albert Einstein College of Medicine, Bronx, New York (Stupak).

Author Contributions: Dr Demesh had full access to all of the data in the study and takes responsibility for the integrity of the data and the accuracy of the data analysis.

Study concept and design: Demesh, Hsueh, Stupak. Acquisition, analysis, or interpretation of data: Demesh, Leonard, Schechter, Dhillon, Hsueh. Drafting of the manuscript: Demesh, Leonard, Schechter, Dhillon, Hsueh.

Critical revision of the manuscript for important intellectual content: Demesh, Leonard, Schechter, Hsueh, Stupak.

Statistical analysis: Demesh, Leonard, Schechter, Dhillon.

Administrative, technical, or material support: Demesh, Dhillon, Hsueh.

Study supervision: Demesh, Hsueh, Stupak.
Conflict of Interest Disclosures: Dr Stupak reports intellectual property for oblique vertical plates, which are related to the devices evaluated in this study, but he has not commercialized the device. No other disclosures are reported.

Meeting Presentations: This study was presented in an abbreviated form at the American Academy of Facial Plastic and Reconstructive Surgery (AAFPRS) meeting at the Combined Otolaryngology Spring Meeting; April 16-18, 2018; National Harbor, Maryland; and at the AAFPRS 12th International Symposium of Facial Plastic Surgery; October 15-18, 2018; Dallas, Texas.

Additional Contributions: Synthetic mandible replicas were provided for our study by Stryker. The company did not receive compensation and was not involved in any data collection or analysis that would affect the integrity of our results. 


\section{REFERENCES}

1. Ellis E III, Miles BA. Fractures of the mandible: a technical perspective. Plast Reconstr Surg. 2007; 120(7)(suppl 2):76S-89S. doi:10.1097/01.prs. 0000260721.74357.e7

2. Ellis E III. Open reduction and internal fixation of combined angle and body/symphysis fractures of the mandible: how much fixation is enough? J Oral Maxillofac Surg. 2013;71(4):726-733. doi:10.1016/j. joms.2012.09.017

3. Barde DH, Mudhol A, Ali FM, Madan RS, Kar S, Ustaad F. Efficacy of 3-dimensional plates over Champys miniplates in mandibular anterior fractures. J Int Oral Health. 2014;6(1):20-26.

4. Mandible-body, simple. AO Foundation. https://www2.aofoundation.org/wps/portal/
surgery?bone=CMF\&segment=Mandible \& classification=91-Body,\%20simple\&showPage= indication. Accessed March 21, 2019.

5. Toma VS, Mathog RH, Toma RS, Meleca RJ. Transoral versus extraoral reduction of mandible fractures: a comparison of complication rates and other factors. Otolaryngol Head Neck Surg. 2003; 128(2):215-219. doi:10.1067/mhn.2003.59

6. Farmand $M$. The 3-D plating system in maxillofacial surgery. J Oral Maxillofac Surg. 1993;51: 166-167.

7. Farmand M. The 3-dimensional plate fixation of fractures and osteotomies. Facial Plast Surg Clin North Am. 1995;3:39-56.

8. Ogundare BO, Bonnick A, Bayley N. Pattern of mandibular fractures in an urban major trauma center. J Oral Maxillofac Surg. 2003;61(6):713-718. doi:10.1053/joms.2003.50118

9. Sadhwani BS, Anchlia S. Conventional $2.0 \mathrm{~mm}$ miniplates versus 3-D plates in mandibular fractures. Ann Maxillofac Surg. 2013;3(2):154-159. doi:10.4103/2231-0746.119231

10. Wusiman P, Yarbag A, Wurouzi G, Mijiti A, Moming A. Three dimensional versus standard miniplate fixation in management of mandibular fractures: a systematic review and meta-analysis. J Craniomaxillofac Surg. 2016;44(10):1646-1654. doi:10.1016/j.jcms.2016.07.027

\title{
Simplifying Mandible Fracture Repair
}

\author{
Clinton D. Humphrey, MD; J. David Kriet, MD
}

Repairing mandible fractures is difficult. Contemporary treatment of a displaced or symptomatic fracture often includes open reduction of the fracture and internal fixation with plates and screws. Rigid or semirigid internal fixation typically elimi-

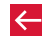

Related article page 271 nates the need for prolonged maxillomandibular fixation, which makes postoperative recovery easier for the patient. Reconstruction plates at least $2 \mathrm{~mm}$ thick are known to reliably withstand mastication forces and stabilize fractures to facilitate bony healing.

However, using bulky reconstruction plates presents several challenges. Manipulating and securing the plate requires broad exposure. Transoral approaches are preferable, but visibility is sometimes too limited for both fracture reduction and plate positioning; open approaches improve exposure but increase operative time, add risks such as facial nerve injury, and prolong patient recovery. Precise placement of a larger reconstruction plate along the inferior mandible border is essential to avoid injuring the inferior alveolar nerve with bicortical screws. Because of these challenges when using larger reconstruction plates, the use of single or multiple miniplates for fixating fractures has become increasingly popular. Minimal contouring is required. Miniplates are easier to visualize and place via transoral approaches, and the monocortical screws used to secure miniplates are less likely to injure the inferior alveolar nerve. Despite these advantages, miniplates also fail more often. Interfragmentary stability is inferior with a miniplate even when the plate is positioned along an ideal line of osteosynthesis. ${ }^{1}$

As an alternative to miniplates, in this issue of JAMA Facial Plastic Surgery, Demesh and colleagues ${ }^{2}$ describe using a 3-dimensional (3-D) vertical box or strut plate with monocortical screws. The 3-D vertical box plate can be placed via limited exposure and with minimal contouring, similar to a miniplate, but with rigidity and resistance to torsion that are comparable with a heavier reconstruction plate. In a retrospective medical record review, Demesh and colleagues found that fracture repair outcomes with the 3-D vertical box plate were comparable to traditional plating techniques; furthermore, operative time, neurosensory deficits, and overall complications were decreased.

When educating novice surgeons about the treatment of mandible fractures, we stress a distinction between loadbearing and load-sharing repairs. Only larger reconstruction plates (of $>2 \mathrm{~mm}$ thickness) can "bear" the load; these plates are accepted as the gold standard for fixating atrophic mandibles, in comminuted fractures, and in other situations where bone stock is greatly decreased or absent. Conversely, the Champy miniplate placed along the superior border to repair an angle fracture is an example of a load-sharing repair; the forces of mastication are shared between the plate and the patient's native bone that the plate is stabilizing. In theory, either a load-bearing or load-sharing repair can be applied to simple fractures in patients with adequate bone stock. Because miniplates are easier to apply, a loadsharing repair would seem to be ideal in most situations. However, a load-bearing repair with a large reconstruction plate-including the time-consuming exposure and increased operative risks-might be selected to treat a simple fracture in a patient with normal bone stock because the patient appears likely to be noncompliant with the required soft diet. The 3-D vertical box or strut plates seem like an ideal alternative for this type of patient.

Although the 3-D vertical box or strut plate is still not considered a load-bearing repair, these newer plates demonstrate a resistance to torsional forces during bone healing similar to reconstruction plates. Perhaps 3-D vertical box or strut plates can "build in the compliance" without the considerable challenges of properly implanting a larger reconstruction plate. The load-sharing vs load-bearing distinction is still important for educating the novice surgeon. But, an experienced facial trauma surgeon often views load bearing to load sharing as a continuum, rather than as distinct categories. Along this continuum, the 3-D vertical box or strut plate feels much 